International Journal of Pure and Applied Mathematics

Volume 96 No. 4 2014, 529-538

ISSN: 1311-8080 (printed version); ISSN: 1314-3395 (on-line version)

url: http://www.ijpam.eu

doi: http://dx.doi.org/10.12732/ijpam.v96i4.8

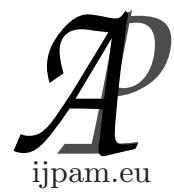

\title{
A NOVEL FOR EXPONENTIAL STABILITY OF \\ LINEAR SYSTEMS WITH MULTIPLE TIME-VARYING DELAYS
}

\author{
Grienggrai Rajchakit \\ Department of Mathematics \\ Maejo University \\ Chiangmai, 50290, THAILAND
}

\begin{abstract}
This paper addresses exponential stability problem for a class of linear systems with multiple time-varying delays. The time delay is any continuous function belonging to a given interval, in which the lower bound of delay is not restricted to zero. By constructing a suitable augmented LyapunovKrasovskii functional combined with Leibniz-Newton's formula, new delaydependent sufficient conditions for the exponential stability of the systems are first established in terms of LMIs.
\end{abstract}

AMS Subject Classification: 15A09, 93D20, 37C75

Key Words: exponential stability, interval multiple time-varying delays, Lyapunov function, linear matrix inequalities

\section{Introduction}

The problem of stability analysis and controller design for time-delay systems have been given considerable attention over the past decades. The existing stabilization results for time delay systems can be classified into two types, 
i.e. delay independent stabilization and delay-dependent stabilization. The delay-independent stabilization provides a controller which stabilizes a system irrespective of the extent of the delay. On the other hand, the delay-dependent stabilization is concerned with the size of the delay which usually provides an upper bound of the delay capable of ensuring the stability for any delay lower than the upper bound. As most physical systems occur in continuous time, consequently the theories for stability analysis and controller synthesis are mainly developed for the continuous time. However, it is more feasible that a discrete-time approach is used for the purpose, as the controller is usually digitally implemented. Stability analysis of linear systems with time-varying delays $\dot{x}(t)=A x(t)+D x(t-h(t))$ is fundamental to many practical problems and has received considerable attention [1-22]. Most of the known results on this problem are derived assuming only that the time-varying delay $h(t)$ is a continuously differentiable function, satisfying some boundedness condition on its derivative: $\dot{h}(t) \leq \delta<1$. In delay-dependent stability criteria, the main concerns is to enlarge the feasible region of stability criteria in given timedelay interval. Interval time-varying delay means that a time delay varies in an interval in which the lower bound is not restricted to be zero. By contracting a suitable argument Lyapunov functional and utilizing free weight matrices, some less conservative conditions for asymptotic stability are derived in [23-28] for systems with time delay varying in an interval. However, the shortcoming of the method used in these works is that the delay function is assumed to be differential and its derivative is still bounded: $\dot{h}(t) \leq \delta$.

This paper gives the improved results for the exponential stability of systems with interval multiple time-varying delays. The time delay is assumed to be a multiple time-varying delays continuous function belonging to a given interval, but not necessary to be differentiable. By constructing argument Lyapunov functional combined with LMI technique, we propose new criteria for the exponential stability of the system.

The paper is organized as follows: Section 2 presents definitions and some well-known technical propositions needed for the proof of the main results. Delay-dependent exponential stability conditions of the system are presented in Section 3.

\section{Preliminaries}

The following notations will be used in this paper. $R^{+}$denotes the set of all real non-negative numbers; $R^{n}$ denotes the $n$-dimensional space with the 
scalar product $\langle.,$.$\rangle and the vector norm \|.\| ; M^{n \times r}$ denotes the space of all matrices of $(n \times r)$-dimensions; $A^{T}$ denotes the transpose of matrix $A ; A$ is symmetric if $A=A^{T} ; I$ denotes the identity matrix; $\lambda(A)$ denotes the set of all eigenvalues of $A ; \lambda_{\min / \max }(A)=\min / \max \{\operatorname{Re} \lambda ; \lambda \in \lambda(A)\} ; x_{t}:=\{x(t+s)$ : $s \in[-h, 0]\},\left\|x_{t}\right\|=\sup _{s \in[-h, 0]}\|x(t+s)\| ; C\left([0, t], R^{n}\right)$ denotes the set of all $R^{n}$-valued continuous functions on $[0, t]$; Matrix $A$ is called semi-positive definite $(A \geq 0)$ if $\langle A x, x\rangle \geq 0$, for all $x \in R^{n} ; A$ is positive definite $(A>0)$ if $\langle A x, x\rangle>0$ for all $x \neq 0 ; A>B$ means $A-B>0$. * denotes the symmetric term in a matrix.

Consider a linear system with interval multiple time-varying delays of the form

$$
\begin{aligned}
& \dot{x}(t)=A x(t)+\sum_{i=1}^{m} D_{i} x\left(t-h_{i}(t)\right), \quad t \in R^{+}, \quad i=1,2, \ldots, m, \\
& x(t)=\phi(t), t \in\left[-h_{2 i}, 0\right], \quad i=1,2, \ldots, m,
\end{aligned}
$$

where $x(t) \in R^{n}$ is the state; $A, D_{i} \in M^{n \times n}, \quad i=1,2, \ldots, m$, and $\phi(t) \in$ $C\left(\left[-h_{2 i}, 0\right], R^{n}\right)$ is the initial function with the norm $\|\phi\|=\sup _{s \in\left[-h_{2 i}, 0\right]} \|$ $\phi(s) \|$; the multiple time-varying delays function $h_{i}(t), i=1,2, \ldots, m$ satisfies the condition

$$
0 \leq h_{0 i} \leq h_{i}(t) \leq h_{1 i}, \quad i=1,2, \ldots, m .
$$

Definition 1. Given $\alpha>0$. The zero solution of system (1) is $\alpha$-exponentially stable if there exist a positive number $N>0$ such that every solution $x(t, \phi)$ satisfies the following condition:

$$
\|x(t, \phi)\| \leq N e^{-\alpha t}\|\phi\|, \quad \forall t \in R^{+} .
$$

We end this section with the following technical well-known propositions, which will be used in the proof of the main results.

Proposition 1. (Cauchy inequality) For any symmetric positive definite matrix $N \in M^{n \times n}$ and $a, b \in R^{n}$ we have

$$
\pm a^{T} b \leq a^{T} N a+b^{T} N^{-1} b .
$$

Proposition 2. [29] For any symmetric positive definite matrix $M \in$ $M^{n \times n}$, scalar $\gamma>0$ and vector function $\omega:[0, \gamma] \rightarrow R^{n}$ such that the integrations concerned are well defined, the following inequality holds

$$
\left(\int_{0}^{\gamma} \omega(s) d s\right)^{T} M\left(\int_{0}^{\gamma} \omega(s) d s\right) \leq \gamma\left(\int_{0}^{\gamma} \omega^{T}(s) M \omega(s) d s\right) .
$$


Proposition 3. [29] Let $E, H$ and $F$ be any constant matrices of appropriate dimensions and $F^{T} F \leq I$. For any $\epsilon>0$, we have

$$
E F H+H^{T} F^{T} E^{T} \leq \epsilon E E^{T}+\epsilon^{-1} H^{T} H
$$

Proposition 4. (Schur complement lemma [29]). Given constant matrices $X, Y, Z$ with appropriate dimensions satisfying $X=X^{T}, Y=Y^{T}>0$. Then $X+Z^{T} Y^{-1} Z<0$ if and only if

$$
\left(\begin{array}{cc}
X & Z^{T} \\
Z & -Y
\end{array}\right)<0 \quad \text { or } \quad\left(\begin{array}{cc}
-Y & Z \\
Z^{T} & X
\end{array}\right)<0
$$

\section{Main Results}

Let us set

$$
\begin{gathered}
\lambda_{1}=\lambda_{\min }(P) \\
\lambda_{2}=\lambda_{\max }(P)+2 h_{2 i} \lambda_{\max }(Q)+2 h_{2 i}^{2} \lambda_{\max }(R)+\left(h_{2 i}-h_{1 i}\right) \lambda_{\max }(U) .
\end{gathered}
$$

Theorem 1. Given $\alpha>0$. The zero solution of the system (1) is $\alpha$ exponentially stable if there exist symmetric positive definite matrices $P, Q, R, U$, and matrices $S_{i}, i=1,2, \ldots, 5$ such that the following $L M I$ holds

$$
\mathcal{M}_{i}=\left[\begin{array}{ccccc}
M_{11 i} & M_{12 i} & M_{13 i} & M_{14 i} & S_{1}-S_{3} A \\
* & M_{22 i} & 0 & M_{24 i} & S_{2} \\
* & * & M_{33 i} & M_{34 i} & S_{3} \\
* & * & * & M_{44 i} & S_{4}-S_{5} \sum_{i=1}^{m} D_{i} \\
* & * & * & * & M_{55 i}
\end{array}\right]<0, \quad i=1,2, \ldots, m
$$

where

$$
\begin{aligned}
M_{11 i} & =A^{T} P+P A+2 \alpha P-\left(e^{-2 \alpha h_{1 i}}+e^{-2 \alpha h_{2 i}}\right) R-S_{1} A+S_{1}+2(Q+R), \\
M_{12 i} & =e^{-2 \alpha h_{1 i}} R-S_{2} A, M_{13 i}=e^{-2 \alpha h_{2 i}} R-S_{3} A, M_{14 i} \\
& =P \sum_{i=1}^{m} D_{i}-S_{1} \sum_{i=1}^{m} D_{i}-S_{3} A
\end{aligned}
$$




$$
\begin{aligned}
& M_{15 i}=S_{1}-S_{3} A, M_{22 i}=-e^{-2 \alpha h_{1 i}}(Q+R), M_{24 i}=S_{2} \sum_{i=1}^{m} D_{i}+e^{-2 \alpha h_{2 i}} U, \\
& M_{33 i}=-e^{-2 \alpha h_{2 i}}(Q+R+U), M_{34 i}=-S_{3} \sum_{i=1}^{m} D_{i}+e^{-2 \alpha h_{2 i}} U \\
& M_{44 i}=S_{4} \sum_{i=1}^{m} D_{i}-e^{-2 \alpha h_{2 i}} U, M_{55 i}=S_{5}+S_{5}^{T}+\left(h_{1 i}^{2}+h_{2 i}^{2}\right) R+\left(h_{2 i}-h_{1 i}\right)^{2} U .
\end{aligned}
$$

Moreover, the solution $x(t, \phi)$ of the system satisfies

$$
\|x(t, \phi)\| \leq \sqrt{\frac{\lambda_{1}}{\lambda_{2}}} e^{-\alpha t}\|\phi\|, \quad \forall t \in R^{+} .
$$

Proof. We consider the following Lyapunov-Krasovskii functional for the system (1)

$$
V\left(t, x_{t}\right)=\sum_{i=1}^{6} V_{i}
$$

where

$$
\begin{aligned}
& V_{1}=x^{T}(t) P x(t), \\
& V_{2}=\int_{t-h_{1 i}}^{t} e^{2 \alpha(s-t)} x^{T}(s) Q x(s) d s \\
& V_{3}=\int_{t-h_{2 i}}^{t} e^{2 \alpha(s-t)} x^{T}(s) Q x(s) d s, \\
& V_{4}=h_{1 i} \int_{-h_{1 i}}^{0} \int_{t+s}^{t} e^{2 \alpha(\tau-t)} \dot{x}^{T}(\tau) R \dot{x}(\tau) d \tau d s, \\
& V_{5}=h_{2 i} \int_{-h_{2 i}}^{0} \int_{t+s}^{t} e^{2 \alpha(\tau-t)} \dot{x}^{T}(\tau) R \dot{x}(\tau) d \tau d s, \\
& V_{6}=\left(h_{2 i}-h_{1 i}\right) \int_{t-h_{2 i}}^{t-h_{1 i}} \int_{t+s}^{t} e^{2 \alpha(\tau-t)} \dot{x}^{T}(\tau) U \dot{x}(\tau) d \tau d s .
\end{aligned}
$$

It easy to check that

$$
\lambda_{1}\|x(t)\|^{2} \leq V\left(t, x_{t}\right) \leq \lambda_{2}\left\|x_{t}\right\|^{2}, \quad \forall t \geq 0,
$$

Taking the derivative of $V_{i}, i=1,2, \ldots, 6$ along the solution of system (1) we 
have

$$
\begin{aligned}
\dot{V}_{1}= & 2 x^{T}(t) P \dot{x}(t) \\
& =2 x^{T}(t)\left[A^{T} P+A P\right] x(t)+2 x^{T}(t) P \sum_{i=1}^{m} D_{i} x\left(t-h_{i}(t)\right) ; \\
\dot{V}_{2}= & x^{T}(t) Q x(t)-e^{-2 \alpha h_{1 i}} x^{T}\left(t-h_{1 i}\right) Q x\left(t-h_{1 i}\right)-2 \alpha V_{2} ; \\
\dot{V}_{3}= & x^{T}(t) Q x(t)-e^{-2 \alpha h_{2 i}} x^{T}\left(t-h_{2 i}\right) Q x\left(t-h_{2 i}\right)-2 \alpha V_{3} ; \\
\dot{V}_{4}= & h_{1 i}^{2} \dot{x}^{T}(t) R \dot{x}(t)-h_{1 i} e^{-2 \alpha h_{1 i}} \int_{t-h_{1 i}}^{t} \dot{x}^{T}(s) R \dot{x}(s) d s-2 \alpha V_{4} ; \\
\dot{V}_{5}= & h_{2 i}^{2} \dot{x}^{T}(t) R \dot{x}(t)-h_{2 i} e^{-2 \alpha h_{2 i}} \int_{t-h_{2 i}}^{t} \dot{x}^{T}(s) R \dot{x}(s) d s-2 \alpha V_{5} ; \\
\dot{V}_{6}= & \left(h_{2 i}-h_{1 i}\right)^{2} \dot{x}^{T}(t) U \dot{x}(t)-\left(h_{2 i}-h_{1 i}\right) e^{-2 \alpha h_{2 i}} \int_{t-h_{2 i}}^{t-h_{1 i}} \dot{x}^{T}(s) U \dot{x}(s) d s-2 \alpha V_{6} .
\end{aligned}
$$

Therefore, we have

$$
\begin{aligned}
& \dot{V}(.)+2 \alpha V(.) \leq \\
& x^{T}(t) M_{11 i} x(t)+2 x^{T}(t)\left[e^{-2 \alpha h_{1 i}} R-S_{2}\right] x\left(t-h_{1 i}\right) \\
& +2 x^{T}(t)\left[e^{-2 \alpha h_{2 i}} R-S_{3} A\right] x\left(t-h_{2 i}\right)+2 x^{T}(t)\left[P \sum_{i=1}^{m} D_{i}\right. \\
& \left.-S_{1} \sum_{i=1}^{m} D_{i}-S_{3} A\right] x(t-h(t)) \\
& +2 x^{T}(t)\left[S_{1}-S_{3} A\right] \dot{x}(t)-x^{T}\left(t-h_{1 i}\right)\left[e^{-2 \alpha h_{1 i}} Q+e^{-2 \alpha h_{1 i}} R\right] x\left(t-h_{1 i}\right) \\
& +2 x^{T}\left(t-h_{1 i}\right)\left[S_{2} \sum_{i=1}^{m} D_{i}+e^{-2 \alpha h_{1 i}} U\right] x\left(t-h_{i}(t)\right)+2 x^{T}\left(t-h_{1 i}\right) S_{2} \dot{x}(t) \\
& -x^{T}\left(t-h_{2 i}\right)\left[e^{-2 \alpha h_{2 i}} Q+e^{-2 \alpha h_{2 i}} R+e^{-2 \alpha h_{2 i}} U\right] x\left(t-h_{2 i}\right) \\
& +2 x^{T}\left(t-h_{2 i}\right) S_{3} \dot{x}(t)+x^{T}\left(t-h_{i}(t)\right)\left[S_{4} \sum_{i=1}^{m} D_{i}-e^{-2 \alpha h_{2 i}} U\right] x\left(t-h_{i}(t)\right) \\
& +2 x^{T}\left(t-h_{i}(t)\right)\left(S_{4}-S_{5} \sum_{i=1}^{m} D_{i}\right) \dot{x}(t) \\
& +\dot{x}^{T}(t)\left[S_{5}+S_{5}^{T}+h_{1 i}^{2} R+h_{2 i}^{2} R+\left(h_{2 i}-h_{1 i}\right)^{2} U\right] \dot{x}(t) \\
& =\zeta_{i}^{T}(t) \mathcal{M}_{i} \zeta_{i}(t),
\end{aligned}
$$

where $\zeta_{i}(t)=\left[x(t), x\left(t-h_{1 i}\right), x\left(t-h_{2 i}\right), x\left(t-h_{i}(t)\right), \dot{x}(t)\right]$. By condition (2), we 
obtain

$$
\dot{V}\left(t, x_{t}\right) \leq-2 \alpha V\left(t, x_{t}\right), \quad \forall t \in R^{+} .
$$

Integrating both sides of (4) from 0 to $t$, we obtain

$$
V\left(t, x_{t}\right) \leq V(\phi) e^{-2 \alpha t}, \quad \forall t \in R^{+} .
$$

Furthermore, taking condition (2) into account, we have

$$
\lambda_{1}\|x(t, \phi)\|^{2} \leq V\left(x_{t}\right) \leq V(\phi) e^{-2 \alpha t} \leq \lambda_{2} e^{-2 \alpha t}\|\phi\|^{2},
$$

then

$$
\|x(t, \phi)\| \leq \sqrt{\frac{\lambda_{2}}{\lambda_{1}}} e^{-\alpha t}\|\phi\|, \quad t \in R^{+},
$$

which concludes the proof by the Lyapunov stability theorem [29].

\section{Conclusion}

In this paper, we have proposed new delay-dependent conditions for the exponential stability of linear systems with non-differentiable interval time-varying delay. Based on the improved Lyapunov-Krasovskii functional and linear matrix inequality technique, the conditions for the exponential stability of the systems have been established in terms of LMIs.

\section{Acknowledgements}

This work was supported by the Thailand Research Fund Grant, the Commission for Higher Education and Faculty of Science, Maejo University, Thailand. The authors thank anonymous reviewers for valuable comments and suggestions, which allowed us to improve the paper.

\section{References}

[1] S.G. Wang, H.S. Yao, Pinning synchronization of the time-varying delay coupled complex networks with time-varying delayed dynamical nodes, Chin.Phys. B 21 (2012) 050508-1-050508-2. 
[2] S. Wang, H. Yao, S. Zheng, Y. Xie, A novel criterion for cluster synchronization of complex dynamical networks with coupling time-varying delays, Commun. Nonlinear Sci. Numer. Simul. 17 (2012) 2997-3004.

[3] Manlika Ratchagit, ON STABILITY OF SWITCHED LINEAR SYSTEMS, International Journal of Pure and Applied Mathematics, Vol. 78 No. 6, 2012, 849-856.

[4] K. Ratchagit, Asymptotic stability of delay-difference system of Hopfield neural networks via matrix inequalities and application, International Journal of Neural Systems, 17(2007), 425-430. DOI: $10.1142 / \mathrm{S} 0129065707001263$

[5] Kreangkri Ratchagit, STABILITY CRITERIA OF LPD SYSTEM WITH TIME-VARYING DELAY, International Journal of Pure and Applied Mathematics, Vol. 78 No. 6, 2012, 857-866.

[6] Kreangkri Ratchagit, STABILITY ANALYSIS OF LINEAR SYSTEMS WITH TIME DELAYS, International Journal of Pure and Applied Mathematics, Vol. 76 No. 1, 2012, 21-28.

[7] Kreangkri Ratchagit , STABILITY OF LINEAR TIME-VARYING SYSTEMS, International Journal of Pure and Applied Mathematics, Vol. 63 No. 4, 2010, 411-417.

[8] Kreangkri Ratchagit , EXPONENTIAL STABILITY OF SWITCHED LINEAR SYSTEMS, International Journal of Pure and Applied Mathematics, Vol. 58 No. 3, 2010, 361-371.

[9] K. Ratchagit , THE SUFFICIENT CONDITIONS FOR STABILITY OF LINEAR TIME-VARYING SYSTEMS WITH STATE DELAYS, International Journal of Pure and Applied Mathematics, Vol. 65 No. 1, 2010, $65-72$.

[10] G. Rajchakit, Stabilization of switched discrete-time systems with convex polytopic uncertainties, Journal of Computational Analysis and Applications 16 (2014) 20-29.

[11] K. Ratchagit and V.N. Phat, Stability criterion for discrete-time systems, J. Ineq. Appl., 2010(2010), 1-6. doi:10.1155/2010/201459

[12] G. Rajchakit, Switching design for the robust stability of nonlinear uncertain stochastic switched discrete-time systems with interval time-varying delay. Journal of Computational Analysis \& Applications 16(2014), 10-19. 
[13] G. Rajchakit, Robust stability and stabilization of nonlinear uncertain stochastic switched discrete-time systems with interval time-varying delays. APPLIED MATHEMATICS and INFORMATION SCIENCES 6(2012), 555-565.

[14] G. Rajchakit, Delay-dependent optimal guaranteed cost control of stochastic neural networks with interval nondifferentiable time-varying delays, ADVANCES IN DIFFERENCE EQUATIONS, 2013(2013), 1-11. DOI: 10.1186/1687-1847-2013-241

[15] K. Ratchagit, A switching rule for the asymptotic stability of discrete-time systems with convex polytopic uncertainties, Asian-European J. Math., 5(2012), 1250025 (12 pages). DOI: 10.1142/S1793557112500258

[16] G. Rajchakit, Stabilization of switched discrete-time systems with convex polytopic uncertainties, Journal of Computational Analysis \& Applications 16(2014), 20-29.

[17] Manlika Rajchakit, Piyapong Niamsup, Grienggrai Rajchakit, A constructive way to design a switching rule and switching regions to mean square exponential stability of switched stochastic systems with non-differentiable and interval time-varying delay, Journal of Inequalities and Applications 2013, 2013:499 doi:10.1186/1029-242X-2013-499

[18] Grienggrai Rajchakit, Delay-Dependent Asymptotical Stabilization Criterion of Recurrent Neural Networks, Applied Mechanics and Materials. 330(2013) 1045-1048. doi:10.4028/www.scientific.net/AMM.330.1045

[19] K. Ratchagit, Asymptotic stability of nonlinear delay-difference system via matrix inequalities and application, International Journal of Computational Methods, pp. 389-397, 2009. DOI: 10.1142/S0219876209001899

[20] M. de la Sen, Global Stability of Polytopic Linear Time-Varying Dynamic Systems under Time-Varying Point Delays and Impulsive Controls, Mathematical Problems in Engineering, vol. 2010, Article ID 693958, 33 pages, 2010. doi: $10.1155 / 2010 / 693958$

[21] G. Rajchakit, Exponential stability of switched linear systems with interval time-varying delays, Proceedings of the 2012 IEEE International Conference on Robotics and Biomimetics December 11-14, 2012, Guangzhou, China, 1502-1506. doi: 10.1109/ROBIO.2012.6491181 
[22] K. Ratchagit, V.N. Phat, Stability and stabilization of switched linear discrete-time systems with interval time-varying delay, Nonlinear Anal. Hybrid Syst. 5 (2011) 605-612. DOI: 10.1016/j.nahs.2011.05.006

[23] VN. Phat, Y. Kongtham, and K. Ratchagit, LMI approach to exponential stability of linear systems with interval time-varying delays, Linear Algebra Appl., Vol. 436, pp. 243-251, 2012. doi: 10.1016/j.laa.2011.07.016

[24] M.S. Mahmoud, Improved Stability and stabilization approach to linear interconnected time-delay systems, Optim. Control Appl. Methods 31 (2010) 81-92.

[25] M.S. Mahmoud, Decentralized reliable control of interconnected systems with time-varying delays, J. Optim. Theory Appl. 143 (2009) 497-518.

[26] P. Niamsup, M. Rajchakit, G. Rajchakit, Guaranteed cost control for switched recurrent neural networks with interval time-varying delay, JOURNAL OF INEQUALITIES AND APPLICATIONS, 2013(2013). DOI: $10.1186 / 1029-242 \mathrm{X}-2013-292$

[27] P. Niamsup, G. Rajchakit, New Results on Robust Stability and Stabilization of Linear Discrete-Time Stochastic Systems with Convex Polytopic Uncertainties, JOURNAL OF APPLIED MATHEMATICS, 2013(2013). DOI: $10.1155 / 2013 / 368259$

[28] G. Rajchakit, Stabilization of switched discrete-time systems with convex polytopic uncertainties, Journal of Computational Analysis and Applications 16 (2014) 20-29.

[29] R.P. Agarwal, Difference Equations and Inequalities, Second Edition, Marcel Dekker, New York, 2000. 